\title{
Liberal Diagnosis and Treatment of Intrauterine Infection Reduces Early-Onset Neonatal Group B Streptococcal Infection but not Sepsis by Other Pathogens
}

\author{
H. Wolf, ${ }^{1 *}$ A.H.P. Schaap, ${ }^{1}$ B.J. Smit, ${ }^{2}$ L. Spanjaard, ${ }^{3}$ and \\ A.H. Adriaanse ${ }^{1}$ \\ ${ }^{1}$ Department of Obstetrics and Gynecology, Academic Medical Center, Amsterdam, The Netherlands \\ ${ }^{2}$ Department of Neonatology, Academic Medical Center, Amsterdam, The Netherlands \\ ${ }^{3}$ Department of Microbiology, Academic Medical Center, Amsterdam, The Netherlands
}

\begin{abstract}
Objective: Comparison of the incidence and case fatality of early-onset group B streptococcus sepsis and sepsis caused by other pathogens in neonates after change of management of intrauterine infection.

Methods: All infants delivered from 1988 through 1997 at a gestational age $\geq 24$ weeks with a birth weight $\geq 500$ gram without lethal congenital abnormalities were eligible for inclusion. Infants delivered by cesarean section before the onset of labor or rupture of membranes were excluded. During the first period (1988-1991) intrauterine infection was diagnosed by a temperature $>38^{\circ} \mathrm{C}$, during the second period (1992-1997) this diagnosis was made at a lower temperature $\left(\geq 37.8^{\circ} \mathrm{C}\right)$ or by fetal tachycardia $\geq 160 / \mathrm{min}$. Treatment of intrauterine infection was similar during both periods with $3 \times 2$ gram amoxicillin and $1 \times 240 \mathrm{mg}$ gentamicin every 24 hours intravenously during labor. Prophylactic treatment during labor was only given to women with a history of an earlier infant with early-onset group B streptococcus sepsis.

Results: During the first period 6,103 infants were included, during the second period 8,504. Intrauterine infection was diagnosed and treated more often in the second period (7.1\% vs. $2.6 \%)$. The incidence of early-onset group B streptococcus sepsis was significantly lower in the second period than in the first period $[0.2 \%$ vs. $0.4 \%$; OR $0.5(0.3-0.9)]$ and survival without disability higher [80\% vs. 52\%; OR 4.5 (1.4-16.5)]. However, in both periods the overall incidence of neonatal sepsis $(3.6 \%$ vs. $3.5 \%)$ and overall mortality because of sepsis $(14.3 \%$ vs.13.1\%) were similar.

Conclusions: Although the early detection of clinical signs of intrauterine infection might have been effective for the prevention of serious sequelae of early-onset group B streptococcus sepsis the overall incidence and mortality from neonatal sepsis remained unchanged. Evaluation of preventive measures for early-onset group B streptococcus sepsis should always take the incidence of neonatal sepsis caused by other pathogens into account. Infect. Dis. Obstet. Gynecol. 8:143-150, 2000. (c) 2000 Wiley-Liss, Inc.
\end{abstract}

KEY WORDS

early-onset neonatal group B streptococcus infection; neonatal sepsis; risk factors; prevention

$\mathrm{T}$ he optimum clinical strategy to prevent neonatal early-onset group B streptococcus infection has not yet been established. Although it is generally assumed that penicillin during labor prevents nearly all cases of early-onset group B streptococcus infection, conclusive statistical evidence is not available. ${ }^{1}$

Three analyses ${ }^{2-4}$ of cost-effectiveness for dif-

*Correspondence to: Dr. H. Wolf, Department of Obstetrics and Gynecology, Room H4-205, Academic Medical Center, PO Box 22660, 1100 DD, Amsterdam, The Netherlands. E-mail: h.wolf@amc.uva.nl 
ferent preventive strategies used comparable risk estimates but different cost calculations and reached different conclusions. Two studies ${ }^{3,4}$ stated that universal recto-vaginal culture at 26 to 28 weeks for group B streptococcus (GBS) and intrapartum treatment of culture-positive high-risk patients in labor was cost effective. A third study ${ }^{2}$ questioned this and concluded that presently either universal treatment during labor, or treatment based on risk factors, or treatment based on preterm delivery and 36-week culture status would be the most effective strategies.

A disadvantage of decision analysis is that the influence of contributing factors is based on research performed some time earlier, while the weight of these factors may well be modified by other changes of management than antibiotic prophylaxis or by technical improvements in neonatal care. Ideally a comparison of different strategies for prevention of early-onset GBS infection should be performed by a randomized trial. Unfortunately, such studies are not feasible due to the low incidence of early-onset GBS infection and consequently the extremely large number of patients that would be required. ${ }^{5}$ Furthermore, most studies of preventive strategies for early-onset GBS infection do not take other causes of neonatal sepsis into account. It is not known presently whether the incidence of neonatal sepsis caused by other organisms is influenced positively or negatively by these strategies. The endpoint of our study is, therefore, not only early-onset GBS infection but also neonatal sepsis of other origin. The purpose of the study is to evaluate a change of management regarding the diagnosis and treatment of women with early signs of intrauterine infection during labor. The hypothesis of this new strategy was that most serious sequelae and death would result from intrauterine onset of early-onset GBS infection, while colonization and onset of infection after delivery would be recognized more easily and could therefore be treated in a more timely manner.

\section{SUBJECTS AND METHODS}

All infants delivered in our tertiary care center from January 1988 through December 1997 at a gestational age $\geq 24$ weeks with a birth weight $\geq 500$ gram without lethal congenital abnormalities were eligible for inclusion in the study. Infants delivered by cesarean section before the onset of labor or rupture of membranes were excluded. Gestational age was calculated by the last menstrual period and early ultrasonographic measuremen, if available.

During the first study period (1988 through 1991) no special preventive measures for earlyonset GBS infection were taken. Intrauterine infection during labor was diagnosed by rectal temperature of $>38.0^{\circ} \mathrm{C}$ on repeated measurement within 30-60 minutes.

During the second period (1992 through 1997) management was based on the hypothesis that signs of intrauterine infection were the major risk factor for early-onset GBS infection. The criteria for suspected intrauterine infection were rectal temperature $\geq 37.8^{\circ} \mathrm{C}$ observed twice within $30-60$ minutes or fetal tachycardia $\geq 160 / \mathrm{min}$.

Treatment for intrauterine infection was similar in both periods with amoxicillin $3 \times 2 \mathrm{gram} / 24 \mathrm{hr}$ and gentamicin $1 \times 240 \mathrm{mg} / 24 \mathrm{hr}$ i.v. until delivery. In case of allergy to penicillin erythromycin $4 \times 1$ $\mathrm{gram} / 24 \mathrm{hr}$ was administered. Prophylactic penicillin during labor was administered only to women with a history of early-onset GBS infection in an earlier infant. Prelabor rupture of membranes was managed expectantly without the use of prophylactic antibiotics. Once antibiotic treatment was started during labor, treatment was continued in the infant(s).

Outcome criteria were neonatal early-onset GBS infection, death or abnormal development of infants after early-onset GBS infection, neonatal sepsis caused by other pathogens, and perinatal mortality. The diagnosis of early-onset GBS infection was confirmed in all cases by a positive culture from material that normally should be sterile (blood, cerebrospinal fluid, or tissue obtained at autopsy). Neonatal sepsis caused by $E$. coli, Staphylococcus species, and other pathogens was diagnosed similarly. Infants with sepsis diagnosed by clinical signs and leukocyte count without confirmation by microbiology were classified separately. Classification of neonatal morbidity was performed by one neonatologist throughout the study period.

Postnatal mortality included all liveborn infants who died before discharge from hospital, irrespective of their age. Follow-up was continued for at least two years in infants after early-onset GBS. Surviving infants were clinically screened and classified as normal or suspected of disability or handicap according to the WHO classification. ${ }^{6}$ 
TABLE I. Demographic and perinatal details of the study population ${ }^{a}$

\begin{tabular}{lcc}
\hline & Period I & $\begin{array}{c}\text { Period II } \\
(1992-1997)\end{array}$ \\
\hline Number of women & $(1988-1991)$ & 8118 \\
Maternal age $(y r)$ & 5833 & $29(12-50)$ \\
Caucasian & $29(12-46)$ & $3984(49.1)$ \\
Primipara & $3280(56.2)$ & $3976(49.0)$ \\
Multiple pregnancy & $2817(48.3)$ & $368(4.5)$ \\
Duration of labor $(\mathrm{hr})$ & $247(4.2)$ & $8(0-218)$ \\
Duration of ruptured membranes (hr) & $8(0-115)$ & $4(0-2615)$ \\
Corticosteroids $>24$ hr & $4(0-2281)$ & $507(6.2)$ \\
Intrauterine infection during labor & $341(5.8)$ & $578(7.1)^{\mathrm{b}}$ \\
Gestational age at delivery (wk) & $150(2.6)^{\mathrm{b}}$ & $40(24-44)$ \\
Number of infants & $40(24-44)$ & 8504 \\
Birth weight (gm) & 6103 & $3200(500-6095)$ \\
Sex male & $3200(515-5320)$ & $4392(51.6)$ \\
Stillborn & $3189(52.3)$ & $21(0.2)$ \\
Neonatal mortality $<4$ wk & $20(0.3)$ & $139(1.6)$ \\
Late mortality 4 wk-l $y r$ & $114(1.9)$ & $9(0.1)$ \\
\hline
\end{tabular}

${ }^{a}$ Values are given as median (range) or number (percentage).

Mantel-Haenszel Chi-square, $P<0.05$.

The incidence of sepsis caused by GBS and other pathogens and perinatal mortality were compared between periods by univariate analysis. Statistical analysis included the Mantel-Haenszel Chi-square test for discrete variables. Odds ratios (OR) are presented with $95 \%$ confidence limits comparing period II versus period I. For continuous variables the two-sample $t$-test or the Wilcoxon rank-sum test were used. Statistical significance was considered at $P<0.05$. Further calculation was performed by stepwise multivariate logistic regression analysis with early-onset GBS infection, disability/handicap or death by early-onset GBS infection and sepsis by other pathogens as dependent variables. Independent variables evaluated in the analysis were study period (second vs. first), maternal age ( $<20,20-34$, and $\geq 35$ years), Caucasian versus non-Caucasian descent, multipara versus nullipara, multi-fetal gestation versus single gestation, diabetes (gestational, grade 2 and 1 ), corticosteroids for neonatal lung maturation before preterm labor, duration of ruptured membranes $(<12$ $\mathrm{hr}, 12-17 \mathrm{hr}, 18-2.3 \mathrm{hr}$, and $\geq 24 \mathrm{hr}$ ), meconium stained amnionic fluid, duration of labor $(<12 \mathrm{hr}, \geq$ $12 \mathrm{hr}$ ), augmentation of labor with oxytocin, duration of second stage $(<0.5 \mathrm{hr}, 0.5-1 \mathrm{hr}$, and $\geq 1 \mathrm{hr}$ ), intrauterine infection during labor, gestational age at delivery [early preterm (24-29 weeks), preterm (30-36 weeks) and term ( $\geq 37$ weeks)], birth weight (500-1499 gram, 1500-2499 gram, and $\geq$ 2500 gram), birth weight $<10$ th percentile of the
Amsterdam birth weight chart, ${ }^{7}$ presentation at birth (cephalic vs. other), and gender (female vs. male). The stepwise logistic regression analysis was performed with BMDP statistical software (Los Angeles, CA). The analysis was started with all variables included in the model. Terms were removed or re-entered at each step by maximum likelihood method. The convergence criterion for the likelihood function and for the parameters was set at 0.0005 .

\section{RESULTS}

During the first period 6,103 infants delivered by 5,833 women were included; during the second period 8,504 infants delivered by 8,118 women. Maternal and neonatal characteristics were comparable between both periods (Table 1). The incidence of diagnosed intrauterine infection during labor was significantly higher in the second period $(7.1 \%)$ than in the first period (2.6\%) [OR 2.9 (2.4-3.5)], demonstrating the effect of the change of definition.

Table 2 demonstrates differences of the incidence of early-onset GBS sepsis and sepsis caused by other pathogens between both periods, subdivided for gestational age. The incidence of earlyonset GBS infection was significantly lower during the second period $(0.2 \%)$ than during the first period $(0.4 \%)$ [OR $0.5(0.3-0.9)$ ]. Case fatality and total mortality from early-onset GBS infection were lower during the second period [OR $0.4(0.1-1.3)$ 
TABLE 2. Gestational age, occurrence of early-onset group B streptococcus (EOGBS) sepsis, sepsis by other pathogens, and mortality of infants delivered during period I and during period II

\begin{tabular}{|c|c|c|c|c|c|c|}
\hline \multirow{2}{*}{\multicolumn{2}{|c|}{$\begin{array}{l}\text { Gestational age } \\
\text { at delivery }\end{array}$}} & \multicolumn{2}{|c|}{ Period I (1988-1991) } & \multicolumn{2}{|c|}{ Period II (1992-1997) } & \multirow{2}{*}{$\begin{array}{l}\text { OR }(95 \% \mathrm{Cl}) \text { of sepsis } \\
\text { Period II vs. Period I }\end{array}$} \\
\hline & & $N(\%)$ & Mortality & $N(\%)$ & Mortality & \\
\hline & All & 397 & $116(29.2)$ & 512 & $149(29.1)$ & \\
\hline \multirow[t]{3}{*}{$24-29 w k$} & EOGBS sepsis & $9(2.3)$ & $4(44.4)$ & $4(0.8)$ & $3(75.0)$ & $0.34(0.1-1.2)$ \\
\hline & Sepsis other pathogen & $122(30.7)$ & $16(13.1)$ & $192(37.5)$ & $32(16.7)$ & $1.35(1.0-1.8)^{\mathrm{b}}$ \\
\hline & All & 816 & $16(2.0)$ & 1114 & $12(1.1)$ & \\
\hline \multirow{3}{*}{$30-36 \mathrm{wk}$} & EOGBS sepsis & $10(1.2)$ & $4(40.0)$ & $8(0.7)$ & $0(-)$ & $0.58(0.2-1.6)$ \\
\hline & Sepsis other pathogen & $55(6.7)$ & $3(5.5)$ & $66(5.9)$ & $4(6.1)$ & $0.87(0.6-1.3)$ \\
\hline & All & 4890 & $8(0.2)$ & 6878 & $8(0.1)$ & \\
\hline \multirow[t]{3}{*}{$\geq 37 \mathrm{wk}$} & EOGBS sepsis & $8(0.2)$ & $2(25.0)$ & $8(0.1)$ & $0(-)$ & $0.71(0.2-2.1)$ \\
\hline & Sepsis other pathogen & $14(0.3)$ & $1(7.1)$ & $16(0.2)$ & $1(6.3)$ & $0.81(0.4-1.8)$ \\
\hline & All & 6103 & $140(2.3)$ & 8504 & $169(2.0)$ & \\
\hline \multirow[t]{2}{*}{ Total } & EOGBS sepsis & $27(0.4)$ & $10(37.0)$ & $20(0.2)$ & $3(15.0)$ & $0.53(0.3-0.9)^{\mathrm{b}}$ \\
\hline & Sepsis other pathogen & $191(3.1)$ & $20(10.5)$ & $274(3.2)$ & $37(13.5)$ & $1.03(0.8-1.3)$ \\
\hline
\end{tabular}

${ }^{a} \bigvee a l u e s$ are given as numbers (percentage) or univariate odds ratio (OR) with $95 \%$ confidence interval $(\mathrm{Cl})$

bMantel-Haenszel Chi-square, $P<0.05$.

and OR $0.2(0.1-0.8)]$. Three infants with earlyonset GBS infection in period I and one in period II were suspected of disability or handicap. Survival without disability/handicap after early-onset GBS infection was higher in the second period [OR 4.5 (1.4-16.5)]. The incidence and outcome of early-onset GBS infection for each year of the study is shown in Fig. 1.

The incidence of neonatal sepsis and the proportion of infants with early-onset GBS infection were strongly influenced by gestational age (test for linear trend, $P<0.0001$ ) (Table 2). In the second period GBS was the causative organism of sepsis in 4 of $196(2 \%)$ of early preterm infants with sepsis, in 8 of $74(11 \%)$ of late preterm infants, and in 8 of 24 (33\%) of term infants, while these figures were $9 / 131(7 \%), 10 / 65(15 \%)$, and $8 / 22(36 \%)$, respectively during the first period.

The incidence of sepsis caused by other pathogens was significantly higher during the second period in early preterm infants [OR 1.35(1.0-1.8)], although the overall incidence was not significantly different between both periods [OR 1.0 (0.8-1.3)] and case fatality [OR $1.3(0.7-2.5)]$ and mortality [OR $1.3(0.7-2.4)]$ due to sepsis by other pathogens were similar. The total incidence of sepsis was $3.6 \%$ during the first and $3.5 \%$ during the second study period [OR $1.0(0.9-1.2)]$, and case fatality [OR $1.0(0.6-1.7)]$ and mortality by sepsis [OR 1.0 (0.6-1.6)] were comparable between periods.

Table 3 subdivides all infants with sepsis for different pathogens. The incidence of sepsis

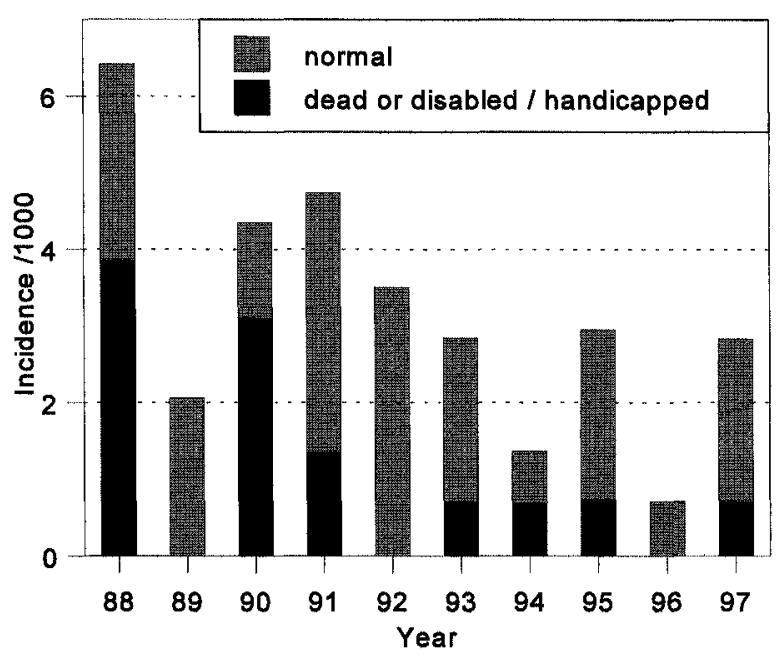

Fig. I. The incidence of early-onset group B streptococcus sepsis in each year of the study period. Infants who recovered and infants who died or remained disabled/ handicapped are presented separately.

caused by $E$. coli was similar between periods, while sepsis by Staphylococcus species and by other pathogens was more frequent during the second period. The number of infants with sepsis diagnosed by clinical signs only, without bacteriological confirmation, was lower in the second period.

Table 4 shows the duration of admission of surviving infants to the neonatal intensive care unit and high care unit. The duration of admission was comparable between infants with early-onset GBS infection and the remaining infants when gestational age at delivery was $<37$ weeks. In term infants a significant difference of the duration of ad- 
TABLE 3. The number and case fatality of all infants in both periods classified as having sepsis caused by group B streptococcus (GBS), Staphylococcus species. Escherichia coli, other pathogens, and infants with clinical sepsis without bacteriological confirmation

\begin{tabular}{|c|c|c|c|c|}
\hline & \multicolumn{2}{|c|}{ Period I (1988-199I) } & \multicolumn{2}{|c|}{ Period II (1991-1997) } \\
\hline & $\mathrm{n}(\%)$ & Case fatality (\%) & $\mathrm{n}(\%)$ & Case fatality (\%) \\
\hline All infants & 6103 & $114(1.9)$ & 8504 & $139(1.6)$ \\
\hline Neonatal sepsis & $218(3.6)$ & $30(13.8)$ & $294(3.5)$ & $40(13.6)$ \\
\hline GBS & $27(0.4)^{a}$ & $10(37.0)$ & $20(0.2)^{a}$ & $3(15.0)$ \\
\hline Staphylococcus & $36(0.6)^{b}$ & $1(2.8)$ & $90(1.1)^{b}$ & $5(5.6)$ \\
\hline E. coli & $8(0.1)$ & $4(50.0)$ & $20(0.2)$ & $5(25.0)$ \\
\hline Other pathogens & $16(0.3)^{c}$ & $5(31.3)$ & $46(0.5)^{c}$ & $13(28.3)$ \\
\hline Clinical sepsis & $13 \mid(2.1)^{d}$ & $10(7.6)$ & $118(1.4)^{d}$ & $14(11.9)$ \\
\hline
\end{tabular}

a,b,c,d Mantel-Haenszel Chi-square, $P<0.05$.

TABLE 4. Duration of neonatal intensive and high care for surviving infants according to gestational age, occurrence of early onset group B streptococcus (EOGBS) sepsis, sepsis by other pathogens, and period ${ }^{\mathrm{a}}$

\begin{tabular}{|c|c|c|c|}
\hline & & \multicolumn{2}{|c|}{ Hospital days } \\
\hline \multicolumn{2}{|c|}{$\begin{array}{l}\text { Gestational age } \\
\text { at delivery }\end{array}$} & $\begin{array}{c}\text { Period I } \\
(1988-1991)\end{array}$ & $\begin{array}{c}\text { Period II } \\
(1992-1997)\end{array}$ \\
\hline & EOGBS sepsis & $37(8-64)^{b}$ & $10(4-25)^{b}$ \\
\hline \multirow[t]{3}{*}{$24-36 w k$} & Sepsis other pathogen & $36(7-143)$ & $45(5-154)$ \\
\hline & Other infants & $8(0-192)$ & $6(0-130)$ \\
\hline & EOGBS sepsis & $13(6-26)^{c}$ & $9(4-16)^{c}$ \\
\hline \multirow{2}{*}{$\geq 37$ wk } & Sepsis other pathogen & $8(4-33)$ & $12(5-26)$ \\
\hline & Other infants & $0(0-99)^{d}$ & $0(0-95)^{d}$ \\
\hline
\end{tabular}

${ }^{a}$ Values are given as median (range).

$b, c, d$ Wilcoxon rank-sum test $P<0.05$

mission was observed between infants with earlyonset GBS infection and the remaining infants. Furthermore, duration of admission of preterm infants with early-onset GBS sepsis was significantly shorter during the second period.

The results of the multivariate regression analysis of those factors that contributed significantly to the predicting model for early-onset GBS infection, for death or disability/handicap by early-onset GBS infection, and for sepsis caused by other pathogens are shown in Table 5. The factors that had significant influence on the incidence of early-onset GBS infection were study period (second $<$ first period), ruptured membranes $(18-23 \mathrm{hr}>0-18 \mathrm{hr}$ or $\geq 24$ $\mathrm{hr}$ ), intrauterine infection during labor and gestational age (early preterm $>$ preterm $>$ term). The only significant factors influencing death or disability/handicap by early-onset GBS infection were study period and gestational age. Risk factors for sepsis caused by other pathogens differed from those for early-onset GBS infection: early preterm gestational age was an extremely strong risk factor, intrauterine infection during labor and birth weight $<10$ th percentile were of minor importance, while study period and duration of ruptured membranes did not influence the model.

\section{DISCUSSION}

Although group B streptococcus infection is generally presented as the leading bacterial cause of disease and death among newborns, this statement applied only to term infants in our study population. Group B streptococcus was the causal pathogen in approximately $35 \%$ of the term infants with sepsis but only in $7 \%$ of all preterm infants with sepsis. Nevertheless, prematurity was a risk factor: $66 \%$ of all infants with early-onset GBS infection were delivered preterm.

The most important factor influencing the incidence of early-onset GBS sepsis is maternal carrier rate. This figure varies largely from $4 \%$ to $40 \%$ (usually 20-30\%), depending on ethnic origin, culture site, and the use of selective culture media. ${ }^{8}$ In the Netherlands a carrier rate during labor of $19 \%$ was reported by selective culture of rectum and introitus. ${ }^{9}$ In our own hospital in 1992 GBS was cultured selectively from rectum and introitus in 41 (27\%) of 150 women during labor. Carrier rate in the Netherlands seems to be comparable to many other countries.

The incidence of early onset GBS sepsis is $0.7-$ $3.7 / 1,000$, depending on geographic area. ${ }^{8}$ The incidence in our population is rather high compared to this figure due to the high incidence of preterm deliveries (19\%) in our tertiary care center as a result of the referral system in the Netherlands. 
TABLE 5. Odds ratio with $95 \%$ confidence limit for early-onset group B streptococcus (EOGBS) sepsis, death or disability/handicap by early-onset group B streptococcus sepsis, and sepsis caused by other pathogens, calculated by the final step of stepwise multivariate logistic analysis ${ }^{\mathrm{a}}$

\begin{tabular}{|c|c|c|c|}
\hline & EOGBS sepsis & $\begin{array}{c}\text { Death or disability/handicap } \\
\text { by EOGBS sepsis }\end{array}$ & $\begin{array}{l}\text { Sepsis caused by } \\
\text { other pathogens }\end{array}$ \\
\hline Period & $0.5(0.3-0.8)$ & $0.2(0.1-0.7)$ & - \\
\hline Multipara & $0.6(0.3-1.2)$ & - & $0.8(0.7-1.0)$ \\
\hline Multiple pregnancy & $0.2(0.1-0.7)$ & - & - \\
\hline Intrauterine infection & $3.3(1.6-6.8)$ & $2.3(0.7-7.7)$ & $1.4(1.1-1.8)$ \\
\hline \multicolumn{4}{|l|}{ Ruptured membranes } \\
\hline$<12 \mathrm{hr}$ & I & - & - \\
\hline $12-17 \mathrm{hr}$ & $1.0(0.2-4.6)$ & & \\
\hline $18-23 \mathrm{hr}$ & $6.6(2.7-16.4)$ & & \\
\hline$\geq 24 \mathrm{hr}$ & $2.5(1.2-5.1)$ & & \\
\hline \multicolumn{4}{|l|}{ Gestational age } \\
\hline$\geq 37$ wk & 1 & 1 & 1 \\
\hline $30-36$ wk & $6.3(3.1-13.0)$ & $7.9(1.8-35.6)$ & $26.3(17.6-39.4)$ \\
\hline $24-29 w k$ & I I.I (4.8-25.9) & $51.3(13.4-196)$ & $205(139-304)$ \\
\hline Birth weight $<$ plo & $0.4(0.1-1.6)$ & - & $1.4(1.0-2.0)$ \\
\hline Gender female vs. male & $0.7(0.4-1.2)$ & $0.5(0.2-1.4)$ & - \\
\hline
\end{tabular}

aFactors marked with - did not contribute significantly to the model.

The extensive literature concerning preventive strategies for neonatal early-onset GBS sepsis was well summarized in a Centers for Disease Control (CDC) recommendation in $1996 .{ }^{10}$ Nine published controlled or randomized studies regarding the efficacy of intrapartum chemoprophylaxis were described, indicating a reduction of neonatal GBS colonization and neonatal early-onset GBS disease. Seven of these studies were accepted by Allen et al. ${ }^{11}$ for meta-analysis. The pooled odds ratio for reduction of early-onset GBS infection was 0.033 (95\% confidence interval 0.0013-0.17) after intrapartum prophylaxis with penicillin. However, Ohlsson et al. ${ }^{1}$ critically appraised the literature and excluded five trials because of duplicate publication, randomization violation, or retrospective data collection. Three of the remaining four trials showed a significant reduction of neonatal group B streptococcal colonization with intrapartum chemoprophylaxis. Although a trend toward a reduction of early-onset GBS infection was reported by these three studies, each lacked the power to reach statistical significance. Ohlsson et al. argued that a meta-analysis was inappropriate due to the heterogeneity of therapeutic interventions and flaws in trial method; and they concluded that intrapartum chemoprophylaxis to reduce early-onset GBS infection was not supported by conclusive evidence. Notwithstanding this, the general opinion is that intrapartum chemoprophylaxis is effective and several preventive strategies have been proposed by professional organizations. ${ }^{10,12-14}$ In 1996 , the CDC developed preventive guidelines in conjunction with experts from relevant disciplines and representatives from professional organizations. They advised that obstetric care practitioners should adopt a strategy for the prevention of early-onset GBS infection and that, presently, prophylaxis based on risk factors or based on preterm delivery and 36-week culture status would be most appropriate. Universal prophylaxis was disclaimed because of risk for the emergence of antimicrobialresistant organisms. Since then substantially more hospitals have adopted preventive policies and surveillance data have revealed a decrease in the incidence of early-onset GBS infection. ${ }^{15,16}$

Rouse et al. ${ }^{2}$ performed an extensive decision analysis evaluating cost-effectiveness of 19 different strategies. They estimated the probability that a pregnant woman would be colonized with GBS as 186:1,000. The probability that a colonized gravida who did not receive intrapartum penicillin would give birth to an infant with early-onset GBS infection was $16: 1,000$ while this probability was $1: 1,000$ if intrapartum penicillin would have been administered (incidence 3/1,000 without and 0.2/1,000 with prophylaxis). These figures were derived from a number of cohort or randomized studies. Costs of rectovaginal cultures were estimated at $\$ 50$ and treatment with ampicillin during labor at $\$ 30$. The sum of charges for treatment of one case of earlyonset GBS infection and its sequelae was estimated 
at $\$ 67,229$ (not including indirect costs). Based on the above estimates Rouse et al. concluded that the most optimum strategies for prevention of earlyonset GBS infection were either universal treatment, or treatment based on risk factors, or treatment based on preterm delivery and 36-week culture status.

The outcome of cost calculations would be different using data from the second period of the present study. Duration of hospitalization of preterm infants was not influenced by the presence of early-onset GBS infection and it is doubtful if costs were increased in these infants. The incidence of early-onset GBS infection in term infants was $1 / 1,000$ with a median duration of hospitalization of 9 days and no long-term sequelae. With these data a screening protocol would not be cost effective.

With adoption of prophylactic strategies against early-onset GBS infection, an increasing number of women will receive antibiotics during labor. However, the consequence of such management on neonatal sepsis caused by other organisms has not been well investigated. Four cases were described with adverse perinatal outcomes from resistant $E n$ terobacteriaceae after antibiotic usage for either PROM or positive group B streptococcal cultures. ${ }^{17}$ Another study ${ }^{18}$ investigated neonatal early-onset $E$. coli sepsis before and after implementation of intrapartum chemoprophylaxis with ampicillin. This study described 30 infants with $E$. coli sepsis. Twelve of 15 infants with an ampicillin-resistant $E$. coli had received antepartum ampicillin, while this occurred only in 1 of 15 infants with an ampicillinsensitive strain. Six of the infants with an ampicillin-resistant strain died, while all of the 15 infants with an ampicillin-sensitive $E$. coli survived. A prospective study ${ }^{19}$ of all cases of neonatal sepsis during a six-year period, when using chemoprophylaxis with ampicillin, described a reduction of early-onset GBS infection while non-group B streptococcal organisms, especially $E$. coli, increased. Both studies support the recent guideline ${ }^{10}$ for the use of penicillin instead of ampicillin. Because penicillin has a narrow spectrum of activity it may be less likely to select for antibiotic resistant organisms, although conclusive evidence for this is not available. Resistance of GBS to penicillin has not been reported, but recently $15 \%$ resistance against clindamycin and $16 \%$ resistance against erythromycin was reported. ${ }^{20}$
Our results demonstrated that early detection of clinical signs of intrauterine infection and early antibiotic treatment resulted in a statistically significant decrease of early-onset GBS infection and reduced the serious sequelae of this infection without large-scale antibiotic administration. Notwithstanding a reduction of early-onset GBS infection the incidence of sepsis caused by other organisms remained unchanged. We should be aware that the prophylactic measures that we take against early-onset GBS infection might result in an increase of sepsis caused by other pathogens. Institutions which adopted antibiotic chemoprophylaxis not only should report on reduced incidence of early-onset GBS infection, but also should register all cases of neonatal sepsis and possible changes of antibiotic resistance of the organisms involved.

\section{REFERENCES}

1. Ohlsson A, Myhr TL. Intrapartum chemoprophylaxis of perinatal group B streptococcal infections: a critical review of randomized controlled trials. Am J Obstet Gynecol 1994;170:910-917.

2. Rouse DJ, Goldenberg RL, Cliver SP, Cutter GR, Mennemeyer ST, Fargason CA Jr. Strategies for the prevention of early-onset neonatal group B streptococcal sepsis: a decision analysis. Obstet Gynecol 1994;83:483494.

3. Yancey MK, Duff P. An analysis of the cost-effectiveness of selected protocols for the prevention of neonatal group B streptococcal infection. Obstet Gynecol 1994; 83:367-371.

4. Mohle-Boetani JC, Schuchat A, Plikaytis BD, Smith JD, Broome CV. Comparison of prevention strategies for neonatal group B streptococcal infection. A populationbased economic analysis. JAMA 1993;270:1442-1448.

5. Landon MB, Harger M, McNellis D, Mercer BM, Thom EA. Prevention of neonatal group B streptococcal infection. Obstet Gynecol 1994;84:460-462.

6. Schaap AH, Wolf H, Bruinse HW, et al. Influence of obstetric management on outcome of extremely preterm growth retarded infants. Arch Dis Child Fetal Neonatal Ed 1997;77:F95-99.

7. Kloosterman GJ. On intrauterine growth. Int J Gynaecol Obstet 1970;8:895-912.

8. Baker CJ, Edwards MS. Group B streptococcal infections. In: Remmington J, Klein JO, editors. Infectious diseases of the fetus and newborn infant. Philadelphia: WB Saunders. 1995. p 980-1054.

9. Adriaanse AH, Kollee LA, Muytjens HL, et al. Randomized study of vaginal chlorhexidine disinfection during labor to prevent vertical transmission of group B streptococci. Eur J Obstet Gynecol Reprod Biol 1995; 61:135-141. 
10. Anonymous. Prevention of perinatal group B streptococcal disease: a public health perspective. Centers for Disease Control and Prevention. MMWR 1996;45:1-24.

11. Allen UD, Navas L, King SM. Effectiveness of intrapartum penicillin prophylaxis in preventing early-onset group B streptococcal infection: results of a metaanalysis. CMAJ 1993;149:1659-1665.

12. Anonymous. ACOG committee opinion. Prevention of early-onset group B streptococcal disease in newborns. Number 173-June 1996. Committee on Obstetric Practice. American College of Obstetrics and Gynecologists. Int J Gynaecol Obstet 1996;54:197-205.

13. Anonymous. Strategies for preventing early onset group B streptococcal infection. Can Commun Dis Rep 1996; 22:44-48.

14. Anonymous. American Academy of Pediatrics Committee on Infectious Diseases and Committee on Fetus and Newborn: Guidelines for prevention of group B streptococcal (GBS) infection by chemoprophylaxis. Pediatrics 1992;90:775-778.

15. Anonymous. Adoption of hospital policies for preven- tion of perinatal group B streptococcal disease-United States, 1997. MMWR Report 1998;47:665-670.

16. Whitney CG, Plikaytis B, Gozansky WS, Wenger JD, Schuchat A. Prevention practices for perinatal group B streptococcal disease: A multi-state surveillance analysis. Obstet Gynecol 1997;89:28-32.

17. McDuffie RS Jr, McGregor JA, Gibbs RS. Adverse perinatal outcome and resistant Enterobacteriaceae after antibiotic usage for premature rupture of the membranes and group B streptococcus carriage. Obstet Gynecol 1993;82:487-489.

18. Joseph TA, Pyati SP, Jacobs N. Neonatal early-onset Escherichia coli disease. The effect of intrapartum ampicillin. Arch Pediatr Adolesc Med 1998;152:35-40.

19. Towers CV, Carr MH, Padilla G, Asrat T. Potential consequences of widespread antepartal use of ampicillin. Am J Obstet Gynecol 1998;179:879-883.

20. Pearlman MD, Pierson CL, Faix RG. Frequent resistance of clinical group B streptococci isolates to clindamycin and erythromycin. Obstet Gynecol 1998;92:258261. 


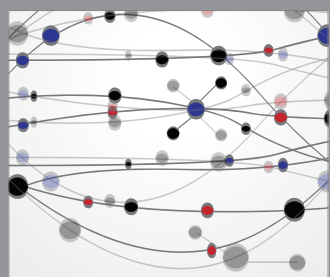

The Scientific World Journal
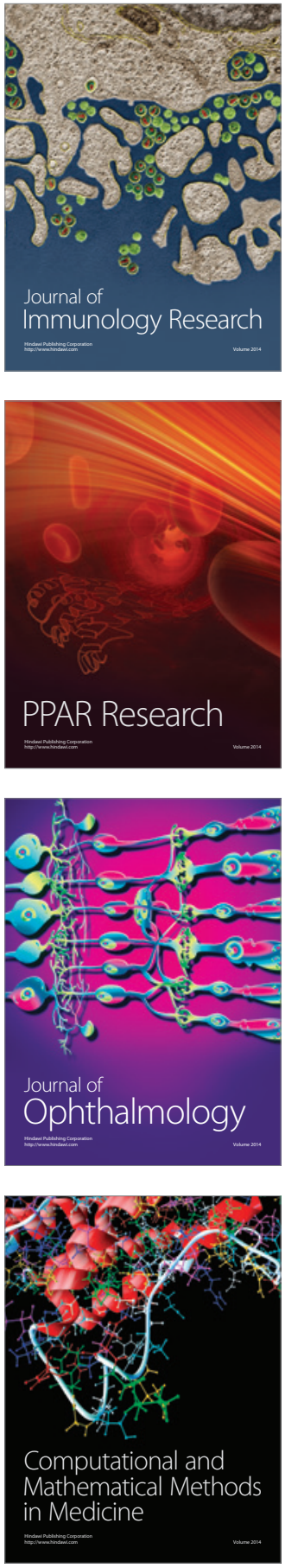

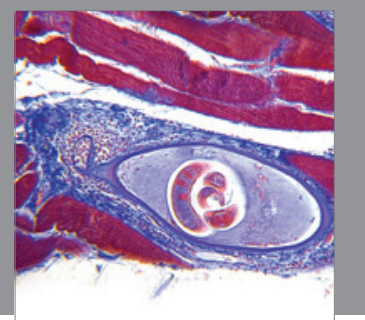

Gastroenterology

Research and Practice
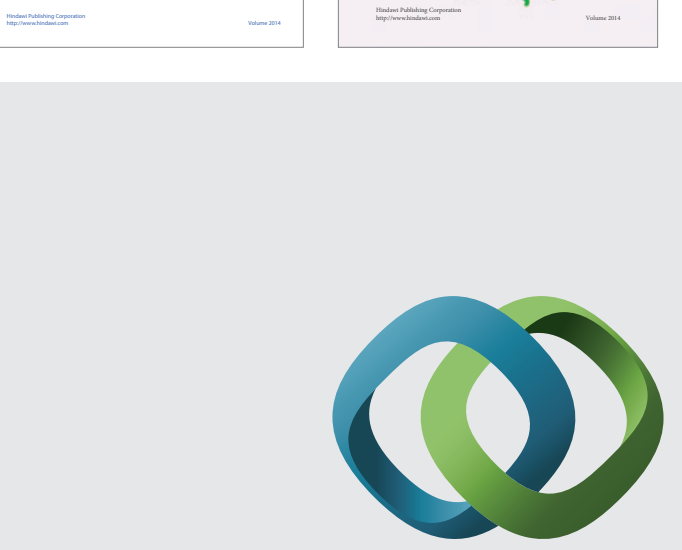

\section{Hindawi}

Submit your manuscripts at

http://www.hindawi.com
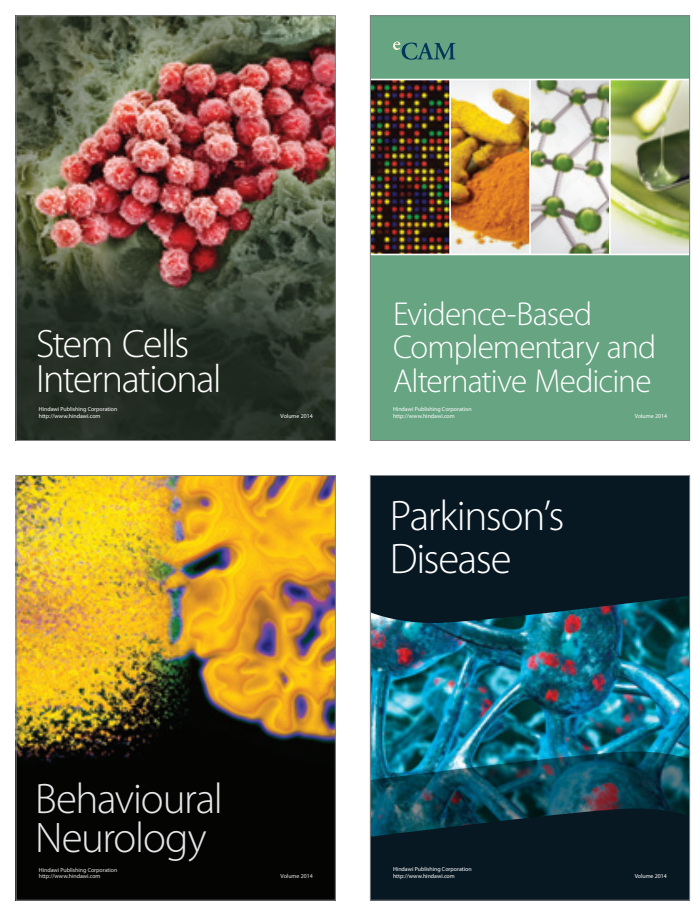

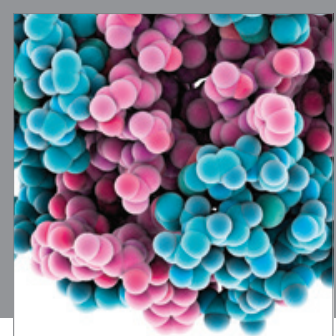

Journal of
Diabetes Research

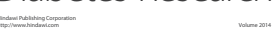

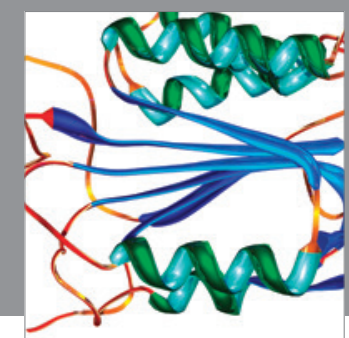

Disease Markers
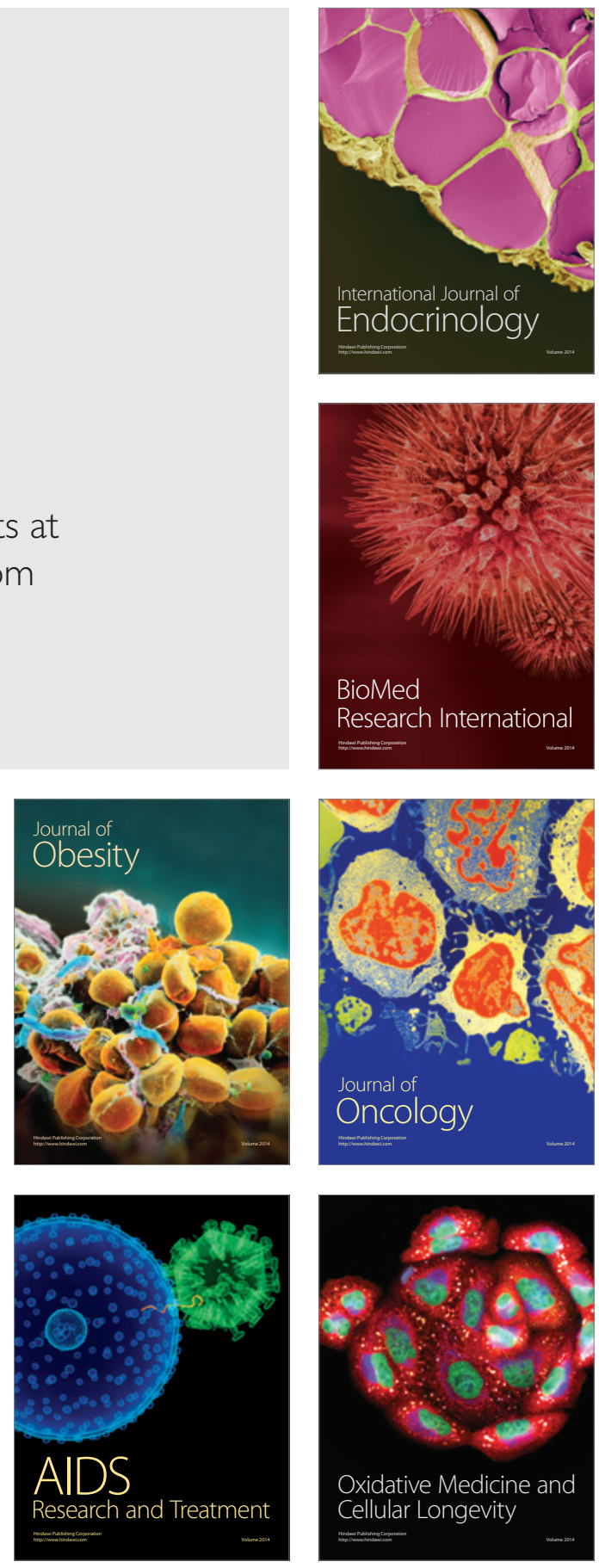